\title{
Shedding Light on the Interfacial Structure of Low-Coverage Alkanethiol Lattices
}

\author{
Evangelina Pensa,* Luis Miguel Azofra, Tim Albrecht, Roberto C. Salvarezza, and Pilar Carro*
}

Cite This: J. Phys. Chem. C 2020, 124, 26748-26758

Read Online

ACCESS | Lill Metrics \& More $\quad$ 回 Article Recommendations

ABSTRACT: A comprehensive description of the self-assembly process of alkanethiols on $\mathrm{Au}(111)$ is presented, focused on the initial formation of the lying down phases. Low-coverage monolayers are prepared by the disintegration of $\mathrm{Au}_{144}(\mathrm{RS})_{60}$ nanoclusters on the reconstructed $(22 \times \sqrt{ } 3)-\mathrm{Au}(111)$ surface. The method provides a limited number of thiols together with a large excess of gold adatoms. Scanning tunneling microscopy and density functional theory calculations were employed to study the transition between low to high thiolate coverage phases. The process involves different lattices and surface transformations, including thiyl radicals on the herringbone reconstruction, radicalinduced herringbone lifting, and the formation of energetically similar metastable phases formed by RS-Au-RS moieties. Results

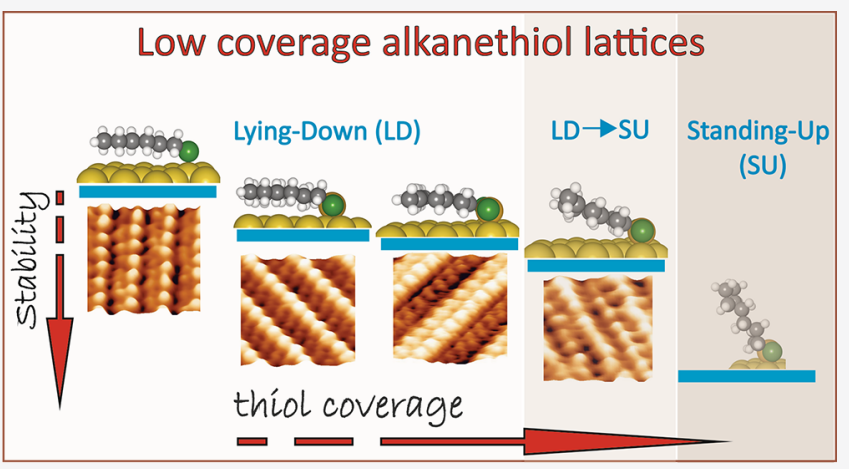
also show that the transition is slow, and different surface structures can coexist on the same sample. Along the process, the first source of $\mathrm{Au}$ adatoms to form the RS-Au-SR moieties is the lifting of the herringbone reconstruction because of the lower energetic cost to extract the extra $\mathrm{Au}$ atom. However, for hexanethiol (and shorter alkanethiols) at low coverage, additional Au adatoms must be taken from terraces leading to vacancy islands. This process can be entirely suppressed by growing the lying down phases in the presence of an excess of $\mathrm{Au}$ adatoms. Taken together, our results shed light on the elusive initial steps of thiol adsorption on clean reconstructed $\mathrm{Au}$, showing that the RS-Au-SR staple motif is also present at the interface of low-coverage self-assembled monolayers.

\section{INTRODUCTION}

The formation of self-assembled monolayers (SAMs) of alkanethiols (RS) on $\mathrm{Au}(111)$ surfaces involves several steps. ${ }^{1,2}$ The process starts with a physisorbed (gas-like) molecular state on the clean reconstructed $(22 \times \sqrt{3}) \mathrm{Au}$ surface ("herringbone" reconstruction). Afterward, the chemisorption of RS molecules takes place in two steps. At low RS concentration, the RS backbones lay parallel to the substrate forming the so-called lying down phases (LD). The RS surface coverage (RS) of LD phases is chain length-dependent, taking values $<0.13 .^{3}$ As RS surface concentration increases, the system grows to dense phases with molecules first in stacked LD phases, ${ }^{4}$ where the interdigitating of hydrocarbons takes place, and finally in standing-up configuration (SU), where hydrocarbon chains are tilted $\alpha \approx 30^{\circ}$ from the substrate normal. The adsorbates arrange into $(\sqrt{3} \times \sqrt{3}) \mathrm{R} 30^{\circ}$ lattices or their $\mathrm{c}(4 \times 2)$ superlattice, both with an RS coverage $\theta_{\mathrm{RS}}=$ $1 / 3 \approx 0.33$. ${ }^{1,2}$

Today, theoretical and experimental evidence supports that RS-Au-SR staples form the $c(4 \times 2)^{5}$ SU lattices, and a mixture of them with RS radicals forms the $(\sqrt{3} \times \sqrt{3}) \mathrm{R} 30^{\circ} \mathrm{SU}$ lattice. $^{6}$ density functional theory (DFT) calculations show that the $c(4 \times 2)$ lattice formed by RS-Au-SR moieties exhibits the best thermodynamic stability compared with their analogues formed by RS radicals or polymer-like alkanethiolate-Au species. ${ }^{5,7,8}$ Experimental evidence arises from scanning tunneling microscopy (STM) images of low-coverage phases $^{9,10}$ and from the structural changes of the substrate after alkanethiol adsorption. The source of $\mathrm{Au}$ adatoms to form the staples is the lifting of the herringbone reconstruction or the $\mathrm{Au}$ atom removal from surface terraces or step edges. Hence, vacancy islands, serrated steps, and the $\mathrm{Au}(111)-(1 \times$ 1) surface are evidence of staple formation. For an SU lattice formed by RS-Au-SR staples, the total amount of adatoms needed is half of the thiol coverage, i.e., $\theta_{\mathrm{Au}}^{\mathrm{ad}}=1 / 6 \approx 0.17$. The herringbone lifting provides $\theta_{\mathrm{Au}}^{\mathrm{ad}}=1 / 23 \approx 0.04$ and the $\mathrm{Au}$ adatom removal the remaining $\theta_{\mathrm{Au}}^{\mathrm{ad}} \approx 0.13$. The latter process generates an equal amount of $\mathrm{Au}$ vacancies that nucleate into islands and became easy to identify and, more importantly, to quantify by STM imaging. Experimental vacancy island

Received: August 20, 2020

Revised: October 16, 2020

Published: November 24, 2020

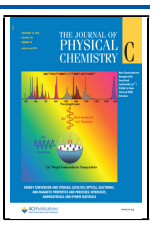


coverage is $\theta_{\mathrm{isl}}^{\mathrm{vac}} \approx 0.12$, in agreement with the expected value. $^{7,11}$

In contrast to the dense SU phases, information about the alkanethiolate species present in the LD phases is much more controversial. There are different methods to prepare LD phases. They include the direct alkanethiol adsorption from the gas phase $\mathrm{e}^{12,13}$ or the controlled desorption of SU phases induced by temperature ${ }^{14-16}$ or an electric field. ${ }^{17}$ In the latter method, the system has a memory of the SU-substrate interaction; hence, vacancy islands or the lifting of the herringbone reconstruction cannot be unambiguously assigned to the LD phase formation. Therefore, preparing $\mathrm{LD}$ phases from the gas phase is the best method to explore the source of $\mathrm{Au}$ adatoms to form alkanethiolate-Au adatom complexes. In this sense, STM images taken during adsorption of hexanethiol from the gas phase reveal domains of LD phases coexisting with the herringbone reconstruction. The appearance of $\mathrm{Au}$ vacancy islands accompanied the growth of the LD phases. ${ }^{12}$ The latter strongly suggests the uptake of Au adatoms from the substrate to form $\mathrm{Au}$ adatom-containing alkanethiol species rather than from the lifting of the herringbone reconstruction. However, a few years later, helium diffraction and STM data show LD phases formed on the herringbone reconstruction with no evidence of vacancy island formation. ${ }^{13}$ Here, RS radicals seem to be present in the $\mathrm{LD}$ domains on terraces, as no source of adatoms is available to form the RS-Au-SR staples. In this context, it is interesting to note that recent density functional calculations (DFT) show that RS radicals or RS-AuSR staples have similar thermodynamic stability. This result opens a question about staples as the thermodynamically stable species, as previously assumed, and the possibility for the coexistence of RS and RS-Au-SR in the LD phases as recently proposed for the $(\sqrt{3} \times \sqrt{3}) \mathrm{R} 30^{\circ}$ SU phase. ${ }^{6}$

In this work, we used a novel method to form $\mathrm{LD}$ phases of alkanethiol SAMs on $\mathrm{Au}(111)$, namely, from the disintegration of $\mathrm{Au}$ nanoclusters (AuNCs). ${ }^{18}$ We have recently shown that the AuNC disintegration transfers mobile RS, RS-Au, and $\mathrm{Au}$ adatoms to the substrate that later arrange into $\mathrm{Au}$ islands and RS SAMs. Importantly, the coverage of the RS monolayer depends on the AuNC size. While $\mathrm{Au}_{25}(\mathrm{RS})_{18}$ decomposition leads to SU RS-SAMs, the $\mathrm{Au}_{144}(\mathrm{RS})_{60}$ disintegrates forming LD phases. Together with this process, a large quantity of gold islands $\theta_{\text {isl }} \approx 0.23$ is formed, reflecting the large excess of $\mathrm{Au}$ adatoms available for the alkanethiolate species during the SAM growth. We use the SR-Au-SR-capped $\mathrm{Au}_{144}(\mathrm{RS})_{60}$ nanoclusters, complemented with conventional thiol adsorption from solution (substrate immersion in RS solution), to form a variety of LD phases on the $(22 \times \sqrt{3})$ reconstructed $\mathrm{Au}(111)$ substrate. By using STM imaging and DFT calculations, we study the corresponding surface structures and their stability, respectively. Results reveal new metastable LD phases that contribute to the understanding of adsorbatesubstrate interactions before the LD-SU phase transition. In particular, the DFT data show the key role of surface chemistry in LD stability, giving a strong support for $\mathrm{Au}$ adatomcontaining alkanethiol species rather than RS radicals. Also, the absence of vacancy islands in our system conclusively shows the relation between RS-Au-SR and vacancy island formation mentioned above. Finally, we propose a pathway consistent with all experimental data reported in the literature to explain the evolution of the LD phases from the physisorbed state on the herringbone reconstruction to the SU phases on the unreconstructed substrate.

\section{METHODS}

General. Glassware was cleaned by immersion in boiling $20 \% \mathrm{HNO}_{3}$ aqueous solution for $\sim 30 \mathrm{~min}$, rinsed with ultrapure water, and dried in an oven at $80^{\circ} \mathrm{C}$. For the AuNC synthesis, an extra cleaning step with aqua regia solution was carried out to remove any metal traces. All reagents and solvents were purchased from Sigma-Aldrich and were used as received (purity, as stated in the text). Ultrapure water (18.2 $\mathrm{M} \Omega \mathrm{cm}$, Purite Select Fusion 160, UK) was employed in all experiments, where required.

AuNC Synthesis and Characterization. The hexanethiolate-capped $\mathrm{Au}_{144}(\mathrm{RS})_{60}$ NCs were synthesized as previously reported, ${ }^{18,19}$ and a full description of the method and characterization can be found in ref 18 . Briefly, $\mathrm{HAuCl}_{4}$. $3 \mathrm{H}_{2} \mathrm{O}(236 \mathrm{mg}, 99.999 \%)$ and tetraoctylammonium bromide (ToABr; $380 \mathrm{mg}, 98 \%)$ were dissolved in methanol $(\mathrm{MeOH}$; $30 \mathrm{~mL}, \geq 99.9 \%)$. After vigorous stirring for $\sim 15 \mathrm{~min}, 1$ hexanethiol (SR; $0.476 \mathrm{~mL}, 95 \%$ ) was added to the mix at room temperature. After $\sim 15 \mathrm{~min}, 6 \mathrm{~mL}$ of $0.5 \mathrm{M}$ fresh $\mathrm{NaBH}_{4}$ $(\geq 98.0 \%)$ solution was rapidly added to the reaction mix under vigorous stirring. The reaction was stopped after $\sim 5 \mathrm{~h}$, and the black precipitate was collected by centrifugation ( 5 $\mathrm{min}$ at $13400 \mathrm{rpm}$ ). Then, the black precipitate was washed with an excess of methanol and collected by centrifugation again. This step was repeated at least 10 times to remove the free thiol residue completely. Afterward, AuNCs were separated from residues of $\mathrm{Au}(\mathrm{I})$-SR polymers by redispersion of the pellet and further mild centrifugation in toluene $(\geq 99.9 \%)$. Finally, acetone $(\geq 99.9 \%)$ was used to separate the $A_{144}(\mathrm{RS})_{60}$ and $\mathrm{Au}_{25}(\mathrm{RS})_{18}$ clusters, as the latter ones are the only one soluble in acetone. After purification, AuNCs were dried and kept at $4{ }^{\circ} \mathrm{C}$ in the dark.

Sample Preparation. Au(111) single crystal disks (99.999\% purity, polished with a roughness of $<0.01 \mu \mathrm{m}$ and an orientation accuracy of $<0.1^{\circ}$, MaTecK GmbH, Germany) were employed in STM measurements. The substrates were cleaned by electropolishing (electrooxidation in $0.1 \mathrm{M} \mathrm{H}_{2} \mathrm{SO}_{4}$ at $5.5 \mathrm{~V}$ using a $\mathrm{Pt}$ counter followed by the dissolution of the $\mathrm{Au}$ oxide layer by dipping in $1 \mathrm{M} \mathrm{HCl}$ ). Afterward, the substrates were annealed in a furnace at $850{ }^{\circ} \mathrm{C}$ for $36 \mathrm{~h}$ (NaberTherm LE 4/11/R6, Germany). Prior to use, the substrates were $\mathrm{H}_{2}$ flame-annealed for ca. $1 \mathrm{~min}$. STM samples were obtained by immersion of Au substrates for $12 \mathrm{~h}$ in $2 \mu \mathrm{M}$ AuNC dispersion. The immersion time was chosen only for practical reasons as it has no effect on either the alkanethiol coverage or the $\mathrm{Au}$ island coverage. ${ }^{18}$ The samples with higher RS coverage were prepared by immersing the $\mathrm{Au}(111)$ substrates in $2 \mu \mathrm{M}$ AuNC dispersion for $12 \mathrm{~h}$ and then for $24 \mathrm{~h}$ in $100 \mu \mathrm{M}$ 1-hexanethiol. Dichloromethane (DCM) was employed as a solvent in all the experiments. The incubation was conducted in the dark and at room temperature $\left(\sim 25^{\circ} \mathrm{C}\right)$ for the time duration stated in the main text. After the incubation period, the samples were rinsed with DCM and dried with $\mathrm{N}_{2}$.

Scanning Tunneling Microscopy. STM measurements were performed using a Keysight 5100 STM (Keysight Technologies, United States). STM tips were prepared by mechanically cutting a $\mathrm{Pt} / \mathrm{Ir}$ wire $(80: 20,0.25 \mathrm{~mm}$ diameter, Goodfellow, UK). STM calibration was performed for each experiment by analyzing both the highly ordered pyrolytic graphite surface $(x-y)$ and Au steps $(z)$. STM images were analyzed with WS $\times$ M software (Nanotec Electronica S.L., 

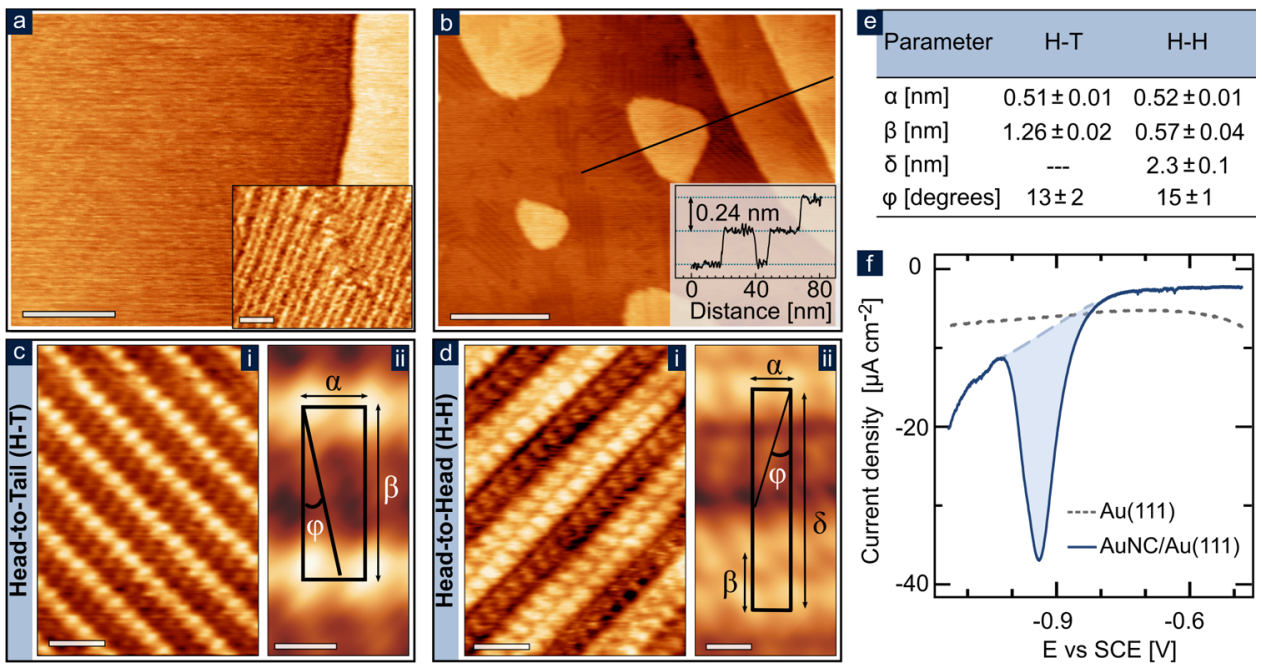

Figure 1. Large-scale STM images of the Au substrate (a) before and (b) after AuNC decomposition. The insets show (a) herringbone reconstruction and (b) height profile along the black line in the image. (c,d) STM images of the different thiolate-containing domains: (c) head-totail and (d) head-to-head. Panels (i) and (ii) show the long-range order of the domains (i) and the unit cells (ii). The latter includes typical parameters describing the unit cell: distances $(\alpha, \beta$, and $\delta)$ and hydrocarbon chain angle with respect to the unit cell $(\varphi)$. (e) Values of the parameters stated in panels (ii). Tunneling parameters used for imaging are $0.5 \mathrm{nA}$ and $0.07 \mathrm{~V}$ (a), $4 \mathrm{nA}$ and $-0.05 \mathrm{~V}(\mathrm{a}$-inset), $0.3 \mathrm{nA}$ and $0.5 \mathrm{~V}$ (b), $0.1 \mathrm{nA}$ and $0.5 \mathrm{~V}$ (c-i,c-ii), and $0.3 \mathrm{nA}$ and $-0.1 \mathrm{~V}$ (d-i,d-ii). Scale bars on the STM images correspond to $30 \mathrm{~nm}(\mathrm{a}, \mathrm{b}), 12 \mathrm{~nm}$ (a-inset), $1.6 \mathrm{~nm}$ (c-i,d-i) and $0.5 \mathrm{~nm}$ (c-ii,d-ii). (f) Typical electroreduction curves of Au(111) substrates before (dotted gray line) and after immersion in AuNC dispersion (full blue line). The RS coverage was obtained by integration of the reduction peak highlighted in light blue.

Spain). ${ }^{20}$ Images were acquired in constant-current mode, with a resolution of $1024 \times 1024$ pixels $^{2}$ and using the tunneling conditions listed into the respective captions. The scan rate was 12.12 lines per second, except for Figures $1 \mathrm{a}, \mathrm{b}$ and $7 \mathrm{a}, \mathrm{b}$ that was 6.06 lines per second. Images were processed with a line-wise flattening to remove tilting effects of the substrate plane and a Gaussian filter to remove the noise.

Electrochemistry. Electrochemical experiments were performed with a $\mathrm{CHI} 760 \mathrm{C}$ potentiostat $(\mathrm{CH}$ Instruments, United States) and a conventional three-electrode glass cell. A $\mathrm{Pt}$ coil and saturated calomel electrode (SCE) served as a counter electrode (CE) and reference electrode (RE), respectively. The Au substrate acted as the working electrode (WE). Electroreduction curves were recorded in a $0.1 \mathrm{M}$ $\mathrm{NaOH}$ (99.99\%) aqueous electrolyte at room temperature $\left(\sim 25^{\circ} \mathrm{C}\right)$ by scanning the potential at $0.1 \mathrm{~V} \mathrm{~s}^{-1}$ in the cathodic direction between -0.2 and $-1.3 \mathrm{~V}$. The electrolyte was degassed with Ar before the measurements, while an Ar atmosphere was kept throughout the experiments. The RS coverage was obtained by integration of the reduction peak, associated to $\mathrm{RS}-\mathrm{Au}+\mathrm{e}^{-} \rightarrow \mathrm{RS}^{-}$. The stated errors are (Bessel-corrected) standard deviations based on $n=6$ independent measurements.

Calculations Based on Density Functional Theory. The calculations were performed using the projector augmented wave (PAW) method, ${ }^{21}$ as implemented in the Vienna ab initio simulation package (VASP). ${ }^{22-24}$ The valence electrons were described within a plane-wave basis set and an energy cutoff of $420 \mathrm{eV}$; the remaining electrons were kept frozen as core states. Electron exchange-correlation was represented by the functional of Perdew, Burke, and Ernzerhof (PBE) of generalized gradient approximation (GGA). ${ }^{25}$ The weak van der Waals forces were treated by the semi-empirical method of Grimme (DFT-D) where the dispersion correction term is added to the conventional Kohn-Sham DFT energy ${ }^{26}$ with the parametrization DFT-D3. ${ }^{27}$ The energy convergence criterion was $10^{-5} \mathrm{eV}$ for self-consistent field energy. The atomic positions were relaxed until the force on the unconstrained atoms was $<10.031 \mathrm{eV} \AA^{-1}$. The calculated $\mathrm{Au}$ lattice constant is $4.099 \AA$, which compares reasonably well with the experimental value $(4.078 \AA))^{28}$ The $\mathrm{Au}(111)$ substrates, both (111) unreconstructed and the herringbone reconstructed, were represented by five atomic layers and a vacuum of $\sim 17 \AA$ that separates two successive slabs in our calculation. Surface relaxation was allowed in the three uppermost $\mathrm{Au}$ layers of the slab, while the atomic coordinates of the adsorbed species were allowed as well to relax without further constraints. Adsorbates were placed just on one side of the slab, and all calculations include a dipole correction. The optimal grid of Monkhorst-Pack, ${ }^{29} k$-points $6 \times 9 \times 1,3 \times 9 \times$ $1,6 \times 7 \times 1,3 \times 7 \times 1,2 \times 4 \times 1,3 \times 5 \times 1$, and $1 \times 9 \times 1$, was used for numerical integration in the reciprocal space of the $(4 \times \sqrt{3})_{\text {rect }}(8 \times \sqrt{3})_{\text {rect }}(4 \times 2 \sqrt{ } 3)_{\text {rect }}(8 \times 2 \sqrt{3})_{\text {rect }}$ $(5 \sqrt{3} \times 2 \sqrt{3}) \mathrm{R} 30^{\circ},(3 \sqrt{3} \times 3)_{\text {rect }}$ and $(22 \times \sqrt{3})_{\text {rect }}$ unit cells, respectively. For gas-phase species, an orthogonal cell of appropriated size was employed. Spin polarization was considered in all gas-phase species.

The RS binding energy $\left(E_{\mathrm{b}}\right)$ is defined as follows

$$
E_{\mathrm{b}}=\frac{1}{N_{\mathrm{RS}}}\left[E_{\mathrm{RS} @ \mathrm{Au}}-E_{\mathrm{Au}}-N_{\mathrm{RS}} E_{\mathrm{RS}}\right]
$$

where $E_{\mathrm{RS} @ A u}, E_{\mathrm{Au}}$ and $E_{\mathrm{RS}}$ are the total energy of the adsorbate-substrate system, the energy of the clean substrate (without RS species), and the adsorbate energy in the gas phase, respectively. $E_{\mathrm{Au}}$ was calculated by allowing only a constrained relaxation of the $\mathrm{Au}$ adatoms because the $\mathrm{Au}$ adatoms in the surface models are in the bridge position, but this site is not a stable minimum. $N_{\mathrm{RS}}$ is the number of RS species per unit cell. A negative number indicates that adsorption is exothermic regarding the separate clean surface and the adsorbate in the gas phase. It is important to note that 
the estimated numerical accuracy in the PBE/DFT-D3 functional used in this work is better than $0.01 \mathrm{eV} .^{27}$

The energy required to lift the herringbone surface $\left(E_{\text {rec }}\right)$ is determined by eq 2 .

$$
E_{\mathrm{rec}}=\frac{1}{2}\left[E_{(22 \times \sqrt{ } 3)}^{\mathrm{R}}-\left(E_{(22 \times \sqrt{3})}^{\mathrm{U}}+2 \mathrm{Au}_{\text {bulk }}\right)\right]
$$

where $E_{(22 \times \sqrt{ } 3)}^{\mathrm{R}}$ and $E_{(22 \times \sqrt{ } 3)}^{\mathrm{U}}$ are the energy of the reconstructed ( 46 atoms in the top layer) and the energy of the unreconstructed (44 atoms in the top layer) of the $(22 \times$ $\sqrt{ }$ 3) unit cell.

The Gibbs free energy of adsorption of each surface structure $(\gamma)$ can be approximated through the total energy from DFT calculations and the unit cell area $(A)$ by using eq 3

$$
\gamma=\frac{N_{\mathrm{RS}}}{A} E_{\mathrm{b}}
$$

Note that here, we focused on electronic energies at equilibrium and zero temperature, i.e., we have neglected vibrational and entropic contributions.

\section{RESULTS AND DISCUSSION}

Figure 1a shows a large-scale STM image of the clean $\mathrm{Au}(111)$ substrate before being immersed in the suspension containing the hexanethiol-capped AuNCs. The substrate exhibits the (22 $\times \sqrt{ } 3$ ) reconstruction known as herringbone structure, which arises because of the compression of atoms in the topmost layer of the crystal. The compression results in extra $4.3 \% \mathrm{Au}$ atoms in the surface layer and leads to a periodic array of fccstacked and hcp-stacked regions separated by $6.4 \pm 0.4 \mathrm{~nm}$ (Figure 1a, inset).

AuNC disintegration takes place after the substrate immersion in the nanocluster suspension. The process leads to the formation of rows of molecules forming ordered domains (Figure $1 \mathrm{~b}$ ) and $\mathrm{Au}$ islands (monoatomic in height, inset in Figure $1 \mathrm{~b}$ ). The herringbone reconstruction and $\mathrm{Au}$ vacancy islands (pits) are absent in these regions of the substrate. The rows of molecules correspond to LD phases. These phases also form by traditional deposition methods, namely, in the gas phase by adsorption of alkanethiols at low pressure $^{12,13}$ or by controlled desorption of SU phases $(\mathrm{c}(4 \times$ $2)$ and $(\sqrt{3} \times \sqrt{3}) \mathrm{R} 30^{\circ}$ with surface coverage $\mathrm{RS} \approx$ $0.33)$. $^{14-17}$

Figure 1c,d shows the two representative LD phases observed in our system. They correspond to the well-known head-to-tail (H-T, Figure 1c) and head-to-head ( $\mathrm{H}-\mathrm{H}$, Figure 1d) configurations. ${ }^{12}$ This historical nomenclature refers to the disposition of the $S$ heads within rows; head-to-head means that two $S$ heads are placed opposite each other, while head-totail highlights that the hydrocarbon chains separate the $S$ heads. Thus, the bright spots in the images (resulting from height topographic and electronic contrast) correspond to the $S$ heads of the alkanethiol species. The $S$ heads form rectangular unit cells with the typical distances and angles listed in Figure 1e. Interestingly, for all the phases, the hydrocarbon backbones are slightly tilted (see $\varphi$ in panels (ii)).

We performed electrochemical measurements to confirm the presence of thiolates and their surface coverage. Figure if shows the electroreduction curves for $\mathrm{Au}(111)$ before and after immersion in AuNC dispersion. The typical reductive desorption peak, absent in the bare substrate and associated to $\mathrm{RS}-\mathrm{Au}+\mathrm{e}^{-} \rightarrow \mathrm{RS}^{-}$, is observed at $0.93 \pm 0.01 \mathrm{~V}$ and has a charge of $24 \pm 3 \mu \mathrm{C} \mathrm{cm}^{-2}$. The latter corresponds to an RS coverage of 0.11 , in good agreement with the theoretical coverage of LD phases $(0.125)$.

Modeling the LD Phases. We have performed DFT calculations to understand the thermodynamic stability of the different $\mathrm{LD}$ phases on the unreconstructed $\mathrm{Au}(111)$ surface. Our experimental data show that the LD phases organize into two different configurations, namely, $\mathrm{H}-\mathrm{T}$ and $\mathrm{H}-\mathrm{H}$ (Figure $1 \mathrm{c}, \mathrm{d})$. Hence, several $\mathrm{H}-\mathrm{T}$ and $\mathrm{H}-\mathrm{H}$ models are proposed to establish the influence of surface chemistry (RS radicals, RSAu-SR staples, and (RS-Au $)_{n}$ polymers) on the lattice's stability. All the proposed models agree with the experimental parameters found in the previous section, i.e., RS coverage and lattice distances and angles. Also, we have extended the study to other surface structures than those in Figure 1 because they have been proposed in the literature or because they differ in subtle details such as relative positions of $S$ heads or hydrocarbon chain orientations (tilted vs parallel). The latter analysis aims to know the impact of geometric parameters on the binding energy of the different species and the thermodynamic stability of their surface structures.

LD Phases Involving RS Thiyl Radicals. Figure 2 shows the optimized surface structures (models I-IV) used to simulate

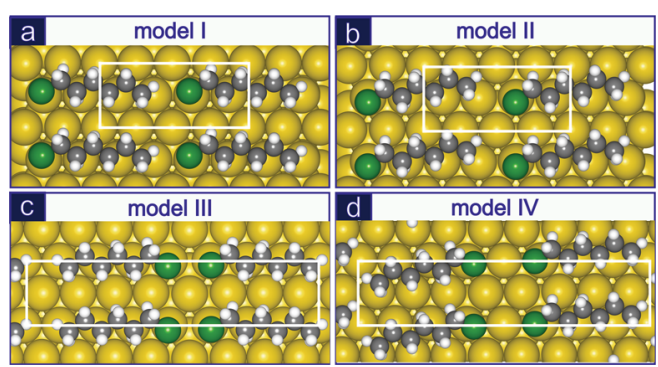

Figure 2. Optimized structures for (a,b) H-T and (c,d) H-H LD phases formed by RS radicals. $(a, b)(4 \times \sqrt{ } 3)_{\text {rect* }}(c, d)(8 \times \sqrt{ } 3)_{\text {rect }}$. The unit cell is outlined in white. Yellow, $\mathrm{Au}$ atom; green, $\mathrm{S}$ atom; gray, $\mathrm{C}$ atom; white, $\mathrm{H}$ atom.

the H-T and H-H LD phases formed by the RS radicals, all of them with $\mathrm{RS}=1 / 8(\approx 0.12)$. Models I and II correspond to the $\mathrm{RS}$ radicals in $\mathrm{H}$-T configuration that can be described by a $(4 \times \sqrt{ } 3)_{\text {rect }}$ unit cell with one thiyl radical (RS) adsorbed with the $S$ head near a top site (Figure 2a,b). For $\mathrm{H}-\mathrm{H}$ configuration, an $(8 \times \sqrt{3})_{\text {rect }}$ lattice is employed with two RS adsorbates per unit cell (models III and IV, Figure 2c,d).

The configuration of the hydrocarbon chains in II/IV models is tilted about $15^{\circ}$ with respect to the $[1 \overline{1} 0]$ Au surface direction, as suggested by the STM images (Figure 1c,d). In contrast, in I/III models, the hydrocarbon chains are parallel and thus inconsistent with the STM images. The parallel hydrocarbon chains introduce an additional inconsistency for $\mathrm{H}-\mathrm{H}$ configuration (model III). The optimization procedure decreases the $S-S$ distance from the initial 0.5 to $0.33 \mathrm{~nm}$ to keep nearly $0.43 \mathrm{~nm}$ between chains (Figure 2c). Therefore, these models are inconsistent with our experimental data (Figure $1 \mathrm{c}, \mathrm{d}$ ) and also with those reported in the literature. ${ }^{10,30}$ The RS binding energy $\left(E_{\mathrm{b}}\right)$ and the surface free energy $(\gamma)$ of the RS models are shown in Table 1.

LD Phases Involving RS-Au-SR Moieties. In models V-XII, the LD phases are built with RS-Au-SR moieties. The H-T configuration is described by a $(4 \times 2 \sqrt{3})_{\text {rect }}$ with one staple per unit cell (Figure $3 a, b)$ or $(8 \times 2 \sqrt{3})_{\text {rect }}$ with two staples 
Table 1. RS Binding Energy $\left(E_{\mathrm{b}}\right)$ and Surface Free Energy $(\gamma)$ for the Different RS Surface Structures ${ }^{a}$

\begin{tabular}{|c|c|c|c|c|c|}
\hline \multirow{2}{*}{$\frac{\text { adsorbate }}{\text { model }}$} & \multicolumn{5}{|c|}{ RS } \\
\hline & configuration & unit cell & $\theta_{\mathrm{RS}}$ & $E_{\mathrm{b}}[\mathrm{eV}]$ & $\gamma\left[\mathrm{meV} \AA^{-2}\right]$ \\
\hline I & $\mathrm{H}-\mathrm{T}$ & $(4 \times \sqrt{ } 3)_{\text {rect }}$ & $1 / 8$ & -2.69 & -46.21 \\
\hline II & $\mathrm{H}-\mathrm{T}$, & $(4 \times \sqrt{ } 3)_{\text {rect }}$ & $1 / 8$ & -2.77 & -47.65 \\
\hline III & $\mathrm{H}-\mathrm{H}$ & $(8 \times \sqrt{ } 3)_{\text {rect }}$ & $2 / 16$ & -2.64 & -45.32 \\
\hline IV & $\mathrm{H}-\mathrm{H}_{/}$ & $(8 \times \sqrt{ } 3)_{\text {rect }}$ & $2 / 16$ & -2.67 & -45.78 \\
\hline
\end{tabular}

${ }^{a} \theta_{\mathrm{RS}}$ is the ratio between the numbers of RS and Au top-layer atoms in the unit cell. Slash shows the models where hydrocarbon chains are tilted with respect to the $[1 \overline{1} 0]$ Au surface direction.

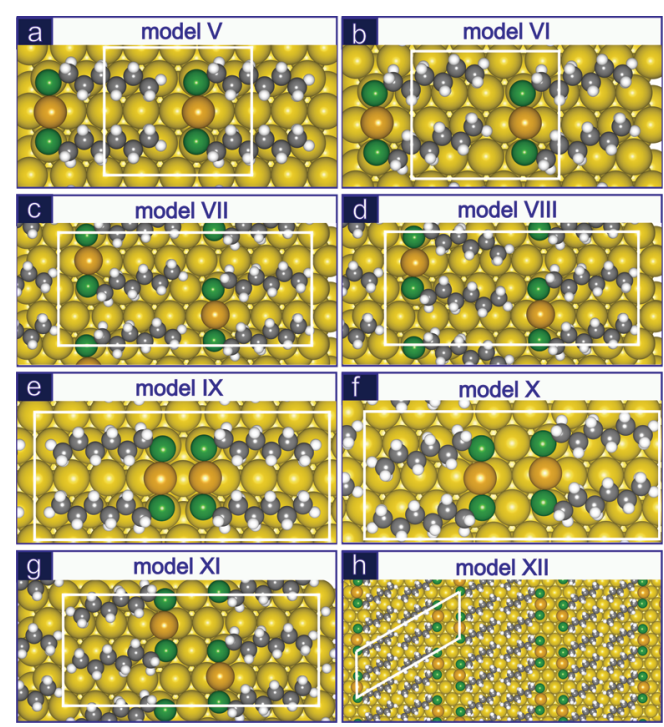

Figure 3. Optimized structures for $(\mathrm{a}-\mathrm{d}) \mathrm{H}-\mathrm{T}$ and $(\mathrm{e}-\mathrm{h}) \mathrm{H}-\mathrm{H}$ phases formed by RS-Au-SR complexes. $(\mathrm{a}, \mathrm{b})(4 \times 2 \sqrt{3})$. $(\mathrm{c}-\mathrm{g})(8 \times$ $2 \sqrt{3})_{\text {rect. }}$ (h) $(5 \sqrt{3} \times 2 \sqrt{3}) \mathrm{R} 30^{\circ}$. The unit cell is outlined in white. Yellow, $\mathrm{Au}$ atom; orange, $\mathrm{Au}$ adatom; green, $\mathrm{S}$ atom; gray, $\mathrm{C}$ atom; white, $\mathrm{H}$ atom.

per unit cell displaced in the vertical axis (Figure 3c,d). The most stable disposition for the staple complex adsorption on the Au surface also implies the $\mathrm{S}$ head on top sites and the $\mathrm{Au}$ adatom placed at a bridge site. The $\mathrm{H}-\mathrm{H}$ phases are described by either $(8 \times 2 \sqrt{3})$ lattices, models IX-XI (Figure $3 \mathrm{e}-\mathrm{g}$ ), or by a $(5 \sqrt{3} \times 2 \sqrt{3}) \mathrm{R} 30^{\circ}$ unit cell, model XII (Figure $\left.3 \mathrm{~h}\right)$. The thiol coverage is $\theta_{\mathrm{RS}}=1 / 8(\approx 0.12)$ for all models except for model XII that is $2 / 15(\approx 0.13)$. The RS binding energy $\left(E_{\mathrm{b}}\right)$ and the surface free energy $(\gamma)$ of the staple models are shown in Table 2.
The major differences between models V-XII (Figure 3ah) are the hydrocarbon chain tilt with respect to the [1]0] Au surface direction and the disposition of the two staple complexes in the unit cell. Also, in this case, the tilted hydrocarbon chain orientation in models VI/VII/VIII/X/XI allows retaining the $\approx 0.5 \mathrm{~nm} \mathrm{~S}-\mathrm{S}$ distance. In contrast, if the hydrocarbon chain is parallel to the $[1 \overline{1} 0]$ direction as in models $\mathrm{V}$ and IX, then the $\mathrm{S}-\mathrm{S}$ distance decreases from the initial 0.5 to $0.33 \mathrm{~nm}$ after the optimization procedure to keep a distance of nearly $0.43 \mathrm{~nm}$ between chains (Figure $3 \mathrm{a}-\mathrm{c}$ ). Therefore, they are inconsistent with our experimental data (Figure 1c,d) and also with those reported in the literature. ${ }^{10,30}$ In model XII, because of the alternate arrangement of the $S$ heads, it is possible to maintain the $S-S$ distance at $0.5 \mathrm{~nm}$ regardless of the tilt of the hydrocarbon chain. In other words, the $\mathrm{H}-\mathrm{H}$ models require tilted hydrocarbon chains to maintain the experimentally observed $\sqrt{3}$ distance between $S$ heads if these $S$ heads are facing each other.

Models XI and XII have the two staple complexes displaced on the vertical axis. However, while in model XI, the $\mathrm{S}$ atoms are aligned, in agreement with the STM (Figure 1d), in model $\mathrm{XII}$, the $\mathrm{S}$ atoms are staggered, a configuration that is not present in our STM images. For the latter model, the hydrocarbon tilt is about $30^{\circ}$, a value higher than the experimentally observed. Despite the discrepancies with the experimental data, model XII has been studied because it is the most stable configuration proposed in the literature for the $\mathrm{H}$ H LD phase of hexanethiolate. ${ }^{30}$

LD Phases Involving RS-Au Polymeric Species. $\mathrm{Au}_{144}(\mathrm{RS})_{60}$ disintegration provides an excess of $\mathrm{Au}$ adatoms and implies the transfer of mobile RS-Au species to the substrate. ${ }^{31,32}$ Hence, we have studied the adsorption of (Au$\mathrm{RS})_{2}$ units in polymeric-like structures forming $\mathrm{H}-\mathrm{H}$ and $\mathrm{H}-\mathrm{T}$ phases. Taking as a starting point models $\mathrm{X}$ and XII, which exhibit similar $E_{\mathrm{b}}$, an extra $\mathrm{Au}$ adatom was added to the respective unit cells forming the corresponding polymeric

Table 2. RS Binding Energy $\left(E_{\mathrm{b}}\right)$ and Surface Free Energy $(\gamma)$ for the Different Staple-Containing Surface Structures ${ }^{a}$

\begin{tabular}{|c|c|c|c|c|c|}
\hline \multirow{2}{*}{$\frac{\text { adsorbate }}{\text { model }}$} & \multicolumn{5}{|c|}{$\mathrm{RS}-\mathrm{Au}-\mathrm{SR}$} \\
\hline & configuration & unit cell & $\theta_{\mathrm{RS}}$ & $E_{\mathrm{b}}[\mathrm{eV}]$ & $\gamma\left[\mathrm{meV} \AA^{-2}\right]$ \\
\hline $\mathrm{V}$ & H-T & $(4 \times 2 \sqrt{3})_{\text {rect }}$ & $2 / 16$ & -3.71 & -63.73 \\
\hline VI & $\mathrm{H}-\mathrm{T} /$ & $(4 \times 2 \sqrt{ } 3)_{\text {rect }}$ & $2 / 16$ & -3.72 & -63.90 \\
\hline VII & $\mathrm{H}-\mathrm{T} /$ & $(8 \times 2 \sqrt{ } 3)_{\text {rect }}$ & $4 / 32$ & -3.73 & -64.08 \\
\hline VIII & $\mathrm{H}-\mathrm{T} /$ & $(8 \times 2 \sqrt{3})_{\text {rect }}$ & $4 / 32$ & -3.72 & -63.95 \\
\hline IX & $\mathrm{H}-\mathrm{H}$ & $(8 \times 2 \sqrt{3})_{\text {rect }}$ & $4 / 32$ & -3.68 & -63.22 \\
\hline $\mathrm{X}$ & $\mathrm{H}-\mathrm{H}$ & $(8 \times 2 \sqrt{ } 3)_{\text {rect }}$ & $4 / 32$ & -3.68 & -63.22 \\
\hline $\mathrm{XI}$ & $\mathrm{H}-\mathrm{H} /$ & $(8 \times 2 \sqrt{3})_{\text {rect }}$ & $4 / 32$ & -3.68 & -63.22 \\
\hline XII & $\mathrm{H}-\mathrm{H}_{/}$ & $(5 \sqrt{3} \times 2 \sqrt{3}) \mathrm{R} 30^{\circ}$ & $4 / 30$ & -3.63 & -66.51 \\
\hline
\end{tabular}

${ }^{a} \theta_{\mathrm{RS}}$ is the ratio between the numbers of RS and Au top-layer atoms in the unit cell. Slash shows the models where hydrocarbon chains are tilted with respect to the $[1 \overline{1} 0]$ Au surface direction. 
species $(\mathrm{RS}-\mathrm{Au})_{2}$. Figure 4 shows the optimized surface structures described by model XIII with an $(8 \times 2 \sqrt{ } 3)_{\text {rect }}$ unit

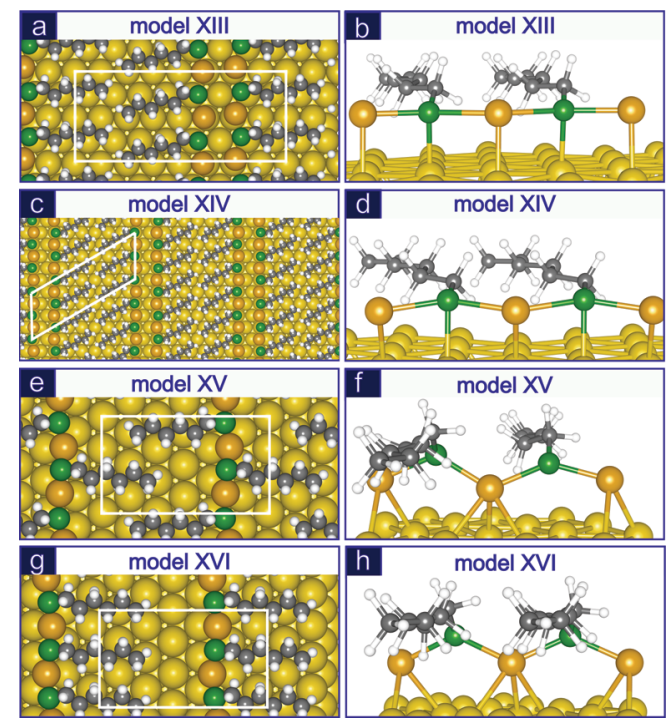

Figure 4. Optimized structures for $(a-d) H-H$ and $(e-h) H-T$ phases using polymeric models. Left and right panels show the top view and the side view of each lattice, respectively. $(a, b)(8 \times \sqrt{ } 3)_{\text {rect }}$ $(\mathrm{c}, \mathrm{d})(5 \sqrt{3} \times 2 \sqrt{3}) \mathrm{R} 30^{\circ}$, and $(\mathrm{e}, \mathrm{f})(3 \sqrt{3} \times 3)_{\text {rect }}$ with $\mathrm{RS}$ in trans configuration and $(\mathrm{g}, \mathrm{h})(3 \sqrt{3} \times 3)_{\text {rect }}$ with $\mathrm{RS}$ in cis configuration. The unit cell is outlined in white. Yellow, Au atom; orange, Au adatom; green, $\mathrm{S}$ atom; gray, $\mathrm{C}$ atom; white, $\mathrm{H}$ atom.

cell (Figure 4a,b) and by model XIV with a $(5 \sqrt{3} \times$ $2 \sqrt{ } 3$ )R $30^{\circ}$ unit cell (Figure $4 \mathrm{c}, \mathrm{d}$ ). In both models, incorporating the extra $\mathrm{Au}$ adatom changes the surface structure considerably. The $\mathrm{S}-\mathrm{Au}$ adatom distance increases from $0.23 / 0.23 \mathrm{~nm}$ (model X/model XII) to $0.27 / 0.26 \mathrm{~nm}$ (model (XIII/model XIV); so, the S-Au adatom bond is weakened. However, this is balanced by the extra bonding associated with a $\mathrm{Au}$ adatom to the surface; as a result, the energetics remains nearly unchanged.

Another strategy to retain the optimal $\mathrm{S}-\mathrm{Au}$ adatom distances in the polymeric structure is to place the polymeric species along the $[1 \overline{1} 0]$ direction instead of along the $[1 \overline{2} 1]$ direction like in models XIII and XIV. Thus, models XV and XVI are described by a $(3 \sqrt{3} \times 3)_{\text {rect }}$ unit cell with the adsorbates placed in trans and cis configurations, respectively. Note that model XV has been already proposed as a model for alkanethiols with intermediate chain length. ${ }^{33}$ Figure 4e,f shows the optimized structure for model XV and Figure $4 \mathrm{~g}$,h for model XVI. In both cases, S-Au adatom distance decreases to $0.24 \mathrm{~nm}$, thus improving the bonding in the polymer structure. However, now, the $S$ heads are far from the $\mathrm{Au}$ surface, thus decreasing their interaction with the substrate (Figure $4 \mathrm{f}-\mathrm{h}$ ). This leads to lower $E_{\mathrm{b}}$ values, which combined with lower surface densities, results in less negative (less stable) $\gamma$ values. Table 3 summarizes the energetic and structural parameters for these polymeric models.

In the following, we discuss the energetics of all the calculated LD phases. Figure 5 summarizes the results shown

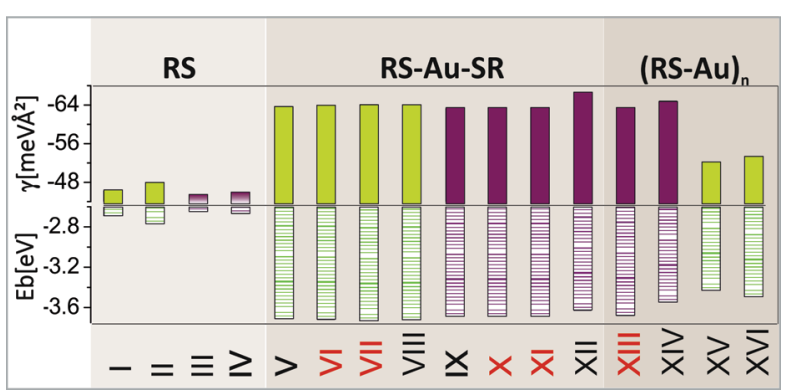

Figure 5. $E_{\mathrm{b}}$ (bottom) and $\gamma$ (top) values for the calculated models in $\mathrm{H}-\mathrm{T}$ (yellow) and $\mathrm{H}-\mathrm{H}$ phases (violet). The panels show the different constitutive moieties present at the interface; from left to right: RS, $\mathrm{RS}-\mathrm{Au}-\mathrm{SR}$, and (RS-Au) $)_{2}$. Red numbers indicate models consistent with the LD phases imaged by STM (Figure 1c,d).

in Tables 1-3. Comparison between the upper left and middle panels shows that $E_{\mathrm{b}}$ values for models where RS units describe the surface chemistry are lower than if RS-Au-SR staples are present. This trend is observed for both $\mathrm{H}-\mathrm{T}$ and $\mathrm{H}-\mathrm{H}$ arrangements. It shows that hexanethiolate molecules are stronger adsorbed to the $\mathrm{Au}(111)$ surface when they are part of the staple motif (RS-Au-SR) rather than if they are directly adsorbed to the substrate (RS). Comparing the models with the $(\mathrm{RS}-\mathrm{Au})_{2}$ polymeric complex (right panel in Figure 5), the ones where $\mathrm{S}$ atoms bind to the $\mathrm{Au}$ adatom in the polymer and also to $\mathrm{Au}$ surface atoms (models XIII and XIV) exhibit $E_{\mathrm{b}}$ values more negative than those where the $S$ heads are far from the substrate surface (models XV and XVI).

To compare the stability of the different surface arrangements, we make use of the surface free energy, $\gamma$. This parameter considers how strong alkanethiol molecules bond to the $\mathrm{Au}$ surface, as reflected by $E_{\mathrm{b}}$ and the molecular density $\left(N_{\mathrm{RS}} / A\right.$, see eq 3). First, all models comprising RS-Au-SR moieties have higher thermodynamic stability, more negative $\gamma$ values, than those involving RS radicals. Within the models formed by RS-Au-SR species, the stability is slightly higher in the $\mathrm{H}-\mathrm{T}$ arrangement (V-VIII) than those in $\mathrm{H}-\mathrm{H}$ models where the $S$ heads are facing each other (IX-XI), thus turning $E_{\mathrm{b}}$ less negative. This is probably because of the repulsion between the adjacent $\mathrm{S}$ heads in this $\mathrm{H}-\mathrm{H}$ arrangement. The exception is model XII that shows the highest thermodynamic stability due to its alternating $S$ heads and to its slightly higher

Table 3. RS Binding Energy $\left(E_{b}\right)$ and Surface Free Energy $(\gamma)$ for the Different H-T and H-H Phases Formed by (RS-Au) Polymeric Surface Structures ${ }^{a}$

\begin{tabular}{|c|c|c|c|c|c|}
\hline \multirow{2}{*}{$\frac{\text { adsorbate }}{\text { model }}$} & \multicolumn{5}{|c|}{$(\mathrm{RS}-\mathrm{Au})_{2}$} \\
\hline & adsorbate & unit cell & $\theta_{\mathrm{RS}}$ & $E_{\mathrm{b}}[\mathrm{eV}]$ & $\gamma\left[\mathrm{meV} \mathrm{\AA ^{-2 } ]}\right.$ \\
\hline XIII & $(\mathrm{RS}-\mathrm{Au})_{2}$ & $(8 \times 2 \sqrt{3})_{\text {rect }}$ & $4 / 32$ & -3.68 & -63.29 \\
\hline XIV & $(\mathrm{RS}-\mathrm{Au})_{2}$ & $(5 \sqrt{3} \times 2 \sqrt{3}) \mathrm{R} 30^{\circ}$ & $4 / 30$ & -3.54 & -64.84 \\
\hline$X V$ & $c i s-(\mathrm{RS}-\mathrm{Au})_{2}$ & $(3 \sqrt{3} \times 3)_{\text {rect }}$ & $2 / 18$ & -3.42 & -52.20 \\
\hline $\mathrm{XVI}$ & trans-(RS-Au $)_{2}$ & $(3 \sqrt{3} \times 3)_{\text {rect }}$ & $2 / 18$ & -3.49 & -53.29 \\
\hline
\end{tabular}

${ }^{a} \theta_{\mathrm{RS}}$ is the ratio between the numbers of RS and Au top-layer atoms in the unit cell. 
coverage $\left(\theta_{\mathrm{RS}}=2 / 15 \approx 0.13\right)$ and therefore a higher $N_{\mathrm{RS}} / A$ value. Finally, we also note that the extra $\mathrm{Au}$ adatom in the polymeric models (XIII and XIV) does not improve the stability compared to the corresponding RS-Au-SR models (X and XII). For models XV and XVI, we found that the lower stability ascribes to the increase in $\mathrm{S}-\mathrm{Au}$ surface distance.

To sum up, for all proposed models, the energetic analysis shows that LD models are slightly sensitive to the lattice unit or geometric parameters such as hydrocarbon chain orientation. Contrarily, they are strongly sensitive to the surface chemistry, i.e., the units present at the interface (RS, $\mathrm{RS}-\mathrm{Au}-\mathrm{SR}$ staple motif, or (RS-Au) $)_{2}$ polymeric complex). In this sense, the models that better reflect the LD phases shown in Figure 1c,d are models VII and VIII for the H-T phase and models X, XI, and XIII for the H-H phase (Figure 5). Note that model XII, which exhibits the highest stability (most negative $\gamma$ value), has not been observed in our experimental images. A plausible explanation is that this phase requires a high coverage, not reached from the disintegration of the AuNCs; so, the system freezes in slightly less stable LD phases. Note that the disintegration process is controlled by other energetic balances, as involve the rupture of AuNCs and the formation of new entities (RS-monolayer and $\mathrm{Au}$ islands). ${ }^{34}$

The Role of Au Adatoms in the Formation of the LD Phases. An important finding in our system is the absence of vacancy islands (Figure $1 \mathrm{~b}$ ). These features are fingerprints of alkanethiolate monolayers at high coverage $\left(\theta_{\mathrm{RS}} \approx 0.33, \theta_{\mathrm{isl}}^{\mathrm{vac}} \approx\right.$ 0.12.) and a strong support of RS-Au-SR moieties at the dense SU phases on the $\mathrm{Au}(111)$. However, for the system under study here, the absence of the vacancy islands can be easily explained when $\mathrm{Au}$ adatoms originated by the AuNC disintegration are considered. The RS-Au-SR LD phases grow under an excess of $\mathrm{Au}$ adatoms, in line with the conventional view on the alkanethiol monolayer formation. $5,7,10,12$

The situation is more controversial for the formation of the LD phases grown from direct adsorption of alkanethiols from the gas phase on clean $(22 \times \sqrt{ } 3)$ Au reconstructed substrates. STM images have shown that the LD phases of adsorbed hexanethiol on the reconstructed surface evolve, creating vacancy islands on the terraces. ${ }^{12}$ This surface evolution is reasonable as the herringbone's lifting provides up to $\theta_{\mathrm{Au}}^{\mathrm{ad}} \approx$ 0.04 , a figure lower than the $\theta_{\mathrm{Au}}^{\mathrm{ad}} \approx 0.06$ needed for the LD phases made up of RS staple complexes ${ }^{9}\left(\theta_{\mathrm{RS}} \approx 0.12\right)$. In contrast, the $\theta_{\mathrm{Au}}^{\mathrm{ad}} \approx 0.04$ provided by the herringbone's lifting should be enough to form LD phases of longer alkanethiols, i.e., when the alkanethiol length increases, the RS coverage decreases and the required $\mathrm{Au}$ adatoms to form the RS-Au-SR species also decreases. However, dodecanethiol LD phases have been observed on the reconstructed surface without evidence of vacancy islands and therefore without an apparent source of $\mathrm{Au}$ adatoms. ${ }^{13,35}$ This result may be explained considering that in that case, the LD phase is still formed by adsorbed RS radicals. Note that the latter implies slow kinetics either for the herringbone lifting or for $\mathrm{Au}$ adatom extraction to form the stable RS-Au-SR moieties, both favored by the interaction between the long hydrocarbon chain and the substrate, as discussed further on.

Concerning the first source of $\mathrm{Au}$ adatoms on smooth terraces, our calculations show the relevant role of the herringbone surface. We estimate that the energy cost to expel one gold atom from the $(22 \times \sqrt{3})$ reconstructed substrate to form a RS-Au-SR staple is $E_{\text {rec }}=+0.34 \mathrm{eV}$. This figure is much lower than that needed to extract an Au adatom from the $\mathrm{Au}(111)(1 \times 1)$ terraces to form the staples, $E_{\mathrm{rec}}=$ $+1.04 \mathrm{eV}$.

Despite the energetic difference between the $\mathrm{Au}$ adatom extraction, the positive $E_{\text {rec }}$ term should be included if LD phases contain RS-Au-SR and are formed using the direct alkanethiol adsorption method. It has been found that RS and RS-Au-SR species in LD phases result in energetically equivalent when $E_{\text {rec }}$ is included, and therefore, they could coexist on the substrate surface. ${ }^{6}$ In contrast, $E_{\mathrm{b}}$ values in Tables 1-3 show that all LD phases containing Au adatoms remain energetically favored by $\approx-1.0 \mathrm{eV} / \mathrm{RS}$ even though the penalty $E_{\text {rec }}=+0.34 \mathrm{eV}$ is included in the calculations. They are always thermodynamically more stable in terms of $\gamma$ than those models formed by the RS radicals. Therefore, they should be the final stable species on the unreconstructed substrate.

Polymeric RS-gold adatom species $(\mathrm{RS}-\mathrm{Au})_{n}$ have been postulated as the basic units of the SU phases in SAMs on $\mathrm{Au}(111) \cdot{ }^{33,36}$ In that case, however, the high energetic cost to provide a large quantity of $\mathrm{Au}$ adatoms $\left(\theta_{\mathrm{Au}}^{\mathrm{ad}} \approx 0.33\right)$ from the substrate turns these SU models unstable compared to the models containing RS-Au-SR, which only need half of that quantity $\left(\theta_{\mathrm{Au}}^{\mathrm{ad}} \approx 0.17\right)$. Similarly, the formation of (RS-Au) species from direct alkanethiol adsorption for the LD phases requires $\theta_{\mathrm{Au}}^{\mathrm{ad}} \approx 0.12$, while only $\theta_{\mathrm{Au}}^{\mathrm{ad}} \approx 0.04$ is provided by the lifting of the herringbone reconstruction. They should also be more unstable than the RS-Au-SR-containing models. However, in our system, there is a large excess of gold adatoms arising from the disintegration of the RS-Au-SR-capped AuNC, a process that involves RS-Au and RS mobile species. ${ }^{31,32}$ Then, $(\mathrm{RS}-\mathrm{Au})_{n} \mathrm{LD}$ phases could also be formed under our experimental conditions, in particular, models XIII and XIV (Table 3). As discussed in the next section, the formation of RS radicals during AuNC disintegration also explains the transient formation of RS domains on our initial reconstructed substrate.

Exploring the Initial Steps: RS Adsorption on Herringbone Reconstruction. We have explored the initial steps of chemisorption where the RS radicals adsorb on the $\mathrm{Au}(111)-(22 \times \sqrt{3})$ reconstructed substrate and then evolve to the more stable RS-Au-SR surface structures on the $\mathrm{Au}(111)-(1 \times 1)$ substrate (Tables $1-3)$. Specifically, we analyze a singular but interesting domain far away from the $\mathrm{Au}$ islands. The domain is formed by rows of bright spots in H-T configuration (Figure 6a) that is described by a rectangular unit cell with a central spot (cf. Figure $6 a$ and its caption for the unit cell descriptor values).

The unit cell for this singular lattice is incommensurable with the unreconstructed $\mathrm{Au}(111)$ substrate. The distances between spots in the unit cell make it impossible to describe this lattice taking the $\mathrm{Au}(111)-(1 \times 1)$ substrate as a reference. However, the $\mathrm{Au}(111)-(22 \times \sqrt{ } 3)$ reconstructed surface, where the $\mathrm{Au}-\mathrm{Au}$ distance in the closed-packed [1히 direction is a $10 \%$ smaller, allows to explain the surface structure observed in the STM image. Supporting this observation, the adsorbate rows are intersected by larger ones separated by about $7 \mathrm{~nm}$ (Figure 6a, green arrow), a distance close to that observed for the $(22 \times \sqrt{3})$ herringbone reconstruction. Thus, we ascribe this domain to a vestige of initial adsorption stages where RS radicals remain interacting with the reconstructed substrate. 


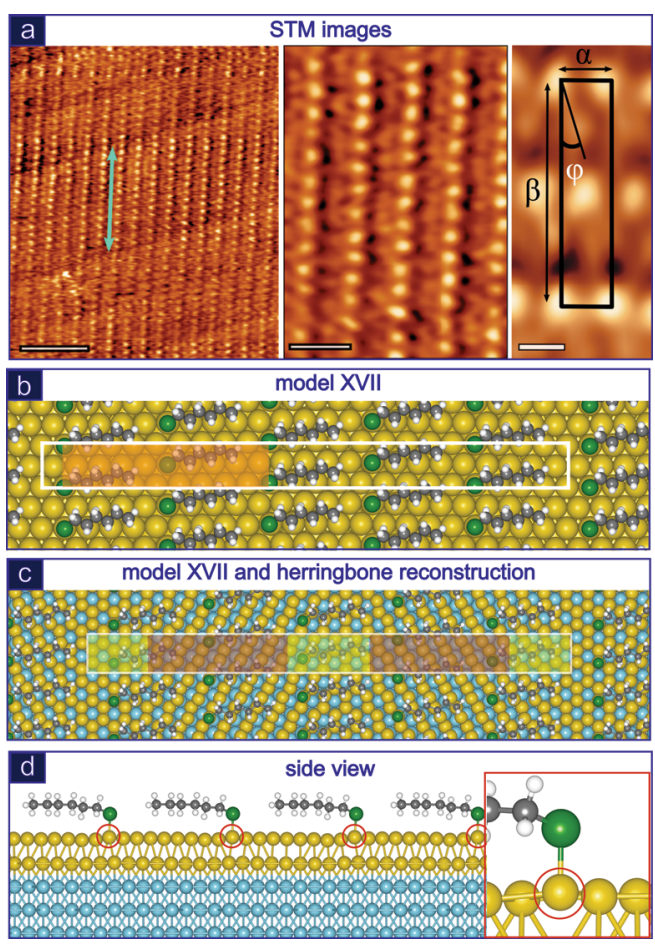

Figure 6. (a) STM images of a singular type of the head-to-tail phase. The left panel shows the long-range order of the domain. The middle and right panels show the detailed structure and the unit cell, respectively. The unit cell descriptors are $\alpha=0.56 \pm 0.01 \mathrm{~nm}, \beta=$ $2.42 \pm 0.01 \mathrm{~nm}$, and $\varphi=17 \pm 3^{\circ}$. Scale bars correspond to $5 \mathrm{~nm}$ (left), $1.6 \mathrm{~nm}$ (middle), and $0.5 \mathrm{~nm}$ (right). The light green arrow in (a) indicates ca. $7.0 \mathrm{~nm}$. Tunneling parameters employed for imaging are $0.3 \mathrm{nA}$ and $0.2 \mathrm{~V}$ for all the images. (b) Optimized structure of RS on $(22 \times \sqrt{3})$ reconstructed $\mathrm{Au}(111)$ (the unit cell observed in the STM images is highlighted in orange). (c) Top layer regions fcc (green) and hcp (orange) (the three bottom layers are colored in blue to visualize the reconstruction). (d) Side view (the Au atoms bonded to $S$ atoms are highlighted in red). The inset shows a zoom-in of image (d). The unit cell is outlined in white. Au atom; green, $\mathrm{S}$ atom; grey, $\mathrm{C}$ atom; white, $\mathrm{H}$ atom.

Figure $6 \mathrm{~b}$ shows the optimized surface structure (model XVII) where the unit cell is formed by five RS $\left(\theta_{\mathrm{RS}}=0.11\right)$ with the $S$ head group sited on top of a Au atom. The RS radicals on this substrate exhibit the weakest adsorption compared with the rest of the models with $E_{\mathrm{b}}=-2.61 \mathrm{eV}$ and the lowest thermodynamic stability since $\gamma=-40.76 \mathrm{meV} \AA^{-2}$. The latter is very similar to those estimated for the RS species on unreconstructed $\mathrm{Au}(111)-(1 \times 1)$ (models I and II in Table 1) although slightly less stable because of the higher coordination of the $\mathrm{Au}$ top-layer atoms. For illustrative purposes, in Figure $6 c$, the three bottom layers have been colored in blue to visualize the herringbone reconstruction. The color code in the unit cell outlines the fcc (pink) and the hcp (cyan) regions of the substrate. Interestingly, the Au atoms attached to $S$ head groups are raised above the surface in approximately $0.25 \AA$ (Figure 6d, inset), twice that the value observed when the RS are adsorbed on the unreconstructed $\mathrm{Au}(111)-(1 \times 1)$ (not shown). This result points out that even the weaker adsorbed RS radical can distort the herringbone reconstruction, favoring in this way the extraction of $\mathrm{Au}$ substrate atoms from the surfaces.

We have estimated the energy balance $\left(\Delta E_{\text {reaction }}\right)$ to lift the herringbone reconstruction using the energetics of the structure shown in Figure 6 as the initial system. The final system comprises the $(8 \times 2 \sqrt{3})_{\text {rect }}$ RS-Au-SR in H-T configuration (model VII) $+(4 \times \sqrt{3})_{\text {rect }}$ RS radical (model II) and a clean Au surface to adjust the mass balance, with both thiol species adsorbed on the unreconstructed $\mathrm{Au}(111)-(1 \times$ 1) surface.

$$
\begin{aligned}
{[5 \mathrm{RS}} & -\mathrm{Au}(111)]_{(22 \times \sqrt{3})} \\
\rightarrow & {[2(\mathrm{RS}-\mathrm{Au}-\mathrm{SR})-\operatorname{Au}(111)]_{(8 \times \sqrt{3})} } \\
& +[\mathrm{RS}-\mathrm{Au}(111)]_{(4 \times \sqrt{3})}+\frac{1}{2}[\mathrm{Au}(111)]_{(4 \times \sqrt{ } 3)}
\end{aligned}
$$

The energetic balance of reaction 4 results in $\Delta E_{\text {reaction }}=$ $-1.72 \mathrm{eV}$, indicating that the lifting of the herringbone reconstruction is thermodynamically favored providing two $\mathrm{Au}$ adatoms per unit cell to form two RS-Au-SR moieties. Thus, the herringbone structure in the same regions of the samples points out that the reaction kinetics (4) is slow. A conclusive explanation for this experimental observation is beyond the scope of the current work. Still, the slow kinetics would be ascribed to one (or more) of the steps involved in the process, namely, collective rearrangement/displacement of the thiol molecules and $\mathrm{Au}(\mathrm{ad})$ atoms and the rupture/formation of new bonds. In line with the above, note that the adsorbed RS radical (model II) should evolve to the more stable RS-Au-SR lattices (Tables 2 and 3) as the RS concentration is increased and more $\mathrm{Au}$ adatoms are removed from the unreconstructed substrate leading to vacancy islands.

Searching for the Bridge: From LD to SU Phases. To determine if the LD phases observed in our system evolve to more dense LD phases when additional hexanethiol molecules are provided, we immerse the samples obtained after AuNC disintegration in hexanethiol containing DCM solution for 24 h. The STM images of these substrates (Figure 7a,b) show tiny vacancy islands (black regions, see arrows in Figure 7 a for eye guidelines) coexisting with disordered rows of molecules (see green arrows in Figure $7 \mathrm{~b}$ for eye guidelines). The Au vacancy islands, absent in the samples prepared by only AuNC disintegration, indicate that additional $\mathrm{Au}$ adatoms have been extracted to form more RS-Au-SR species.

In some regions of the substrate, the rows of molecules organize into small domains with the molecules separated 0.57 $\pm 0.02 \mathrm{~nm}$ along the row and an inter-row distance of $1.10 \pm$ $0.06 \mathrm{~nm}$ (Figure $7 \mathrm{~b}$, inset). The bright spots are displaced $60^{\circ}$ regarding those present in the adjacent row. Interestingly, even if RS are in excess, the system still does not evolve to the SU phase, as it is observed for direct immersion of the bare $\mathrm{Au}(111)$ under the same conditions. We believe that the growing conditions in the current experiment produce an extremely stable LD phase, which acts as kinetic traps on the road to the formation of the standing-up phase; i.e., the LD to SU phase transition takes place on a less defective (aged) LD surface structure. It is very different when the process takes place in one step on more defective domains of LD phases.

Based on the STM image and the stability analysis summarized in Figure 5, we propose a $(2 \sqrt{3} \times 2 \sqrt{3}) \mathrm{R} 30^{\circ}$ RS-Au-SR lattice model in H-T configuration with $\theta_{\mathrm{RS}}=1 / 6 \approx$ 0.17 (Figure $7 \mathrm{c}$ ) and accordingly $\theta_{\mathrm{Au}}^{\mathrm{ad}} \approx 0.08$, thus explaining the formation of vacancy islands in this experiment (model XVIII). Initially, the hydrocarbon chains were placed parallel to the $\mathrm{Au}$ surface, but after optimization, the hydrocarbon chains spontaneously lift from the surface, forming a tilt angle 


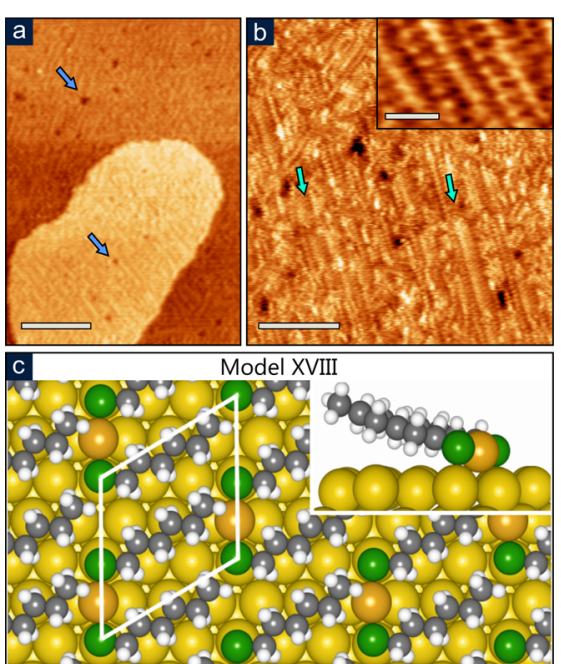

Figure 7. (a,b) Disintegrated AuNC on the $\mathrm{Au}(111)$ sample after immersion in $100 \mu \mathrm{M}$ hexanethiol DCM solution for $24 \mathrm{~h}$. (a) Extensive area of the substrate showing Au islands and small vacancy islands (blue arrows). (b) STM image showing the disordered rows of molecules (green arrows). (inset) High-resolution image of the rows. Scale bars correspond to $20 \mathrm{~nm}$ (a), $10 \mathrm{~nm}$ (b), and $2 \mathrm{~nm}$ (b-inset). Tunneling parameters used for imaging are $0.5 \mathrm{nA}$ and $0.3 \mathrm{~V}(\mathrm{a}), 0.2$ $\mathrm{nA}$ and $-0.3 \mathrm{~V}(\mathrm{~b})$, and $0.3 \mathrm{nA}$ and $0.6 \mathrm{~V}$ (b-inset). (c) Optimized structure of the $(2 \sqrt{3} \times 2 \sqrt{3}) \mathrm{R} 30^{\circ}$ RS-Au-SR model. (inset) sideview detail. The white rectangle corresponds to the unit cell. Yellow, $\mathrm{Au}$ atom; orange, $\mathrm{Au}$ adatom; green, $\mathrm{S}$ atom; gray, $\mathrm{C}$ atom; white, $\mathrm{H}$.

with respect to the surface normal $\alpha=75^{\circ}$. The larger distance from the $\mathrm{Au}$ surface reduces the hydrocarbon chain-substrate interaction, a fact that is reflected in a slight decrease in the binding energy to $E_{\mathrm{b}}=-3.46 \mathrm{eV}$. Conversely, the thermodynamic stability increases to $\gamma \approx-79.13 \mathrm{meV} \AA^{-2}$ due to an increase in the surface coverage $\left(N_{\mathrm{RS}} / A\right.$ term in eq 3).

Interestingly, the $(2 \sqrt{ } 3 \times 2 \sqrt{ } 3) \mathrm{R} 30^{\circ}$ lattice has the same surface area as the $c(4 \times 2)$ unit cell (Figure $8 \mathrm{a}$, middle panel). This suggests that the $(2 \sqrt{3} \times 2 \sqrt{3})$ R $30^{\circ}$ lattice can evolve to the $\mathrm{c}(4 \times 2)$ by simply incorporating one RS-Au-SR staple, i.e., going from $\theta_{\mathrm{RS}} \approx 0.17$ to $\theta_{\mathrm{RS}} \approx 0.33$. In fact, starting with the H-T LD phase described by an $(8 \times 2 \sqrt{3})_{\text {rect }}$ lattice, we can see how the subunit cell $(\sqrt{ } 19 \times \sqrt{19}) \mathrm{R} 23.4^{\circ}$ can easily evolve to the $(2 \sqrt{3} \times 2 \sqrt{3}) \mathrm{R} 30^{\circ}$ by a staple displacement in the $[1 \overline{1} 0]$ direction (red arrow in Figure $8 \mathrm{a}$, left panel).

To incorporate more hexanethiol molecules to the $(2 \sqrt{3} \times$ $2 \sqrt{3}) \mathrm{R} 30^{\circ}$ unit cell (Figure $8 \mathrm{a}$, middle panel), the $\alpha$ value decreases, thus allowing more molecules to reach the substrate surface. After additional $\mathrm{Au}$ adatom extraction and diffusion, the RS-Au-SR adopts the most stable SU phase $\mathrm{c}(4 \times 2)$ with $\theta_{\mathrm{RS}} \approx 0.33$ and $\alpha=33^{\circ}$ (Figure 8a, right panel). A scheme showing the SAM evolution in terms of thermodynamic stability and $\alpha$ values in going from the LD phase-formed RS radicals on the herringbone reconstruction $\left(\theta_{\mathrm{RS}} \approx 0.12\right)$ to the dense SU $\mathrm{c}(4 \times 2)$ phase $\left(\theta_{\mathrm{RS}} \approx 0.33\right)$ on $\mathrm{Au}(111)(1 \times 1)$ formed by RS-Au-SR moieties is depicted in Figure $8 \mathrm{~b}$.

\section{CONCLUSIONS}

By a combination of STM imaging and DFT calculation, we provide a comprehensive description of the hexanethiol LD phases going from the initial chemisorbed step to their transition to the $c(4 \times 2)$ RS-Au-SR SU phase. The LD $\rightarrow$ SU transition involves different surface processes, namely, thiyl

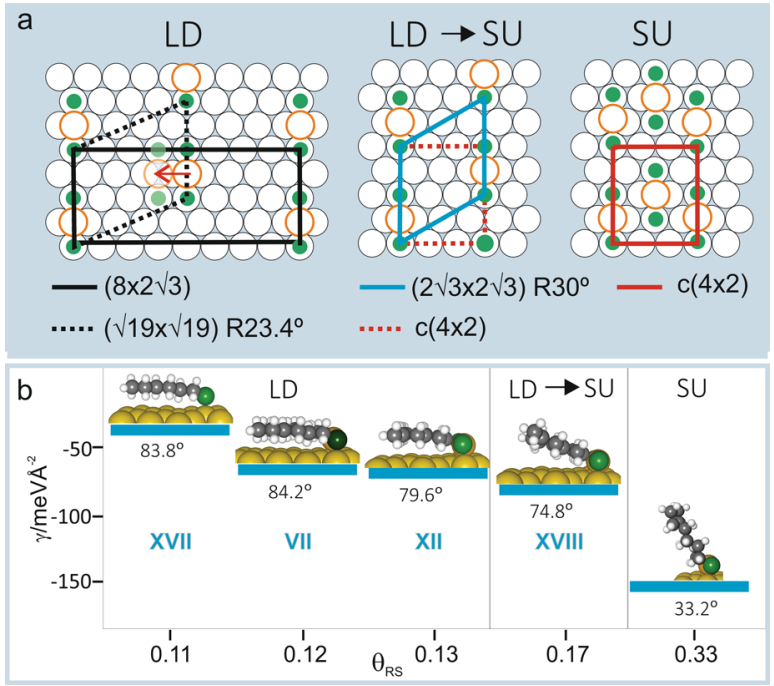

Figure 8. (a) Scheme showing the transformation from the diluted LD staple lattice to that of the dense SU phase. Left panel, $(8 \times 2 \sqrt{3})$ lattice $\left(\theta_{\mathrm{RS}}=1 / 8 \approx 0.12\right)$; middle panel, $(2 \sqrt{3} \times 2 \sqrt{3}) \mathrm{R} 30^{\circ}$ lattice $\left(\theta_{\mathrm{RS}}=1 / 6 \approx 0.17\right)$; right panel, $\mathrm{c}(4 \times 2)$ phase $\left(\theta_{\mathrm{RS}}=1 / 3 \approx 0.33\right)$. The RS-Au-SR position after rearrangement (indicated by the red arrow) is depicted in light colors. (b) Surface free energy $(\gamma)$ as a function of the $\theta_{\mathrm{RS}}$. The corresponding model and the tilt angle are depicted in the graph. Yellow, Au atom; orange, Au adatom; green, $\mathrm{S}$ atom; gray, $\mathrm{C}$ atom; white, $\mathrm{H}$ atom.

radicals on the herringbone reconstruction, radical-induced herringbone lifting, and the formation of energetically similar metastable phases formed by RS-Au-RS moieties. We have also found that thermodynamic stability of the LDs lattices is determined by the surface chemistry (RS, RS-Au-SR, or (RS$\mathrm{Au})_{2}$ ) rather than subtle details in adsorbate configuration (tilt of the lying down hydrocarbon chain or $\mathrm{S}$ adsorption site). As a result, a family of energetically possible RS-Au-SR models in $\mathrm{H}-\mathrm{H}$ or $\mathrm{H}-\mathrm{T}$ configurations is obtained. Results have also shown that transformation from one phase to another, driven by minor differences in the surface free energy, is slow, and different surface structures can coexist on the same sample. Therefore, thermodynamic stability and kinetic factors explain the polymorphism usually observed in this system. We can also conclude that the first source of $\mathrm{Au}$ adatoms to form the RSAu-SR moieties is the lifting of herringbone reconstruction because of the lower energetic cost to extract the $\mathrm{Au}$ atom. Still, for hexanethiol (and shorter alkanethiols) LD phases, additional $\mathrm{Au}$ adatoms must be taken from terraces leading to vacancy islands. However, if the process takes place in excess of $\mathrm{Au}$ adatoms, then vacancy island formation is completely suppressed. ${ }^{34}$ Our results shed light on the elusive initial steps of thiol adsorption on clean reconstructed surfaces.

\section{AUTHOR INFORMATION}

\section{Corresponding Authors}

Evangelina Pensa - Department of Chemistry, Imperial College London, London SW7 2AZ, U.K.; Physics Department, Technical University of Munich, 85748 Garching, Germany; 이이.org/0000-0003-1090-0830; Email: e.pensa@tum.de

Pilar Carro - Área de Química Física, Departamento de Química, Facultad de Ciencias, Universidad de La Laguna, Instituto de Materiales y Nanotecnología, 38200 La Laguna, 
Spain; (1) orcid.org/0000-0001-8073-9857;

Email: pcarro@ull.edu.es

\section{Authors}

Luis Miguel Azofra - Instituto de Estudios Ambientales y Recursos Naturales (i-UNAT), Universidad de Las Palmas de Gran Canaria (ULPGC), Campus de Tafira, 35017 Las Palmas de Gran Canaria, Spain; ํㅏㅇㅣ.org/0000-00034974-1670

Tim Albrecht - Department of Chemistry, Imperial College London, London SW7 2AZ, U.K.; (1) orcid.org/0000-00016085-3206

Roberto C. Salvarezza - Instituto de Investigaciones Fisicoquímicas Teóricas y Aplicadas (INIFTA), Facultad de Ciencias Exactas, Universidad Nacional de La Plata, CONICET, La Plata 1900, Argentina; ㅇorcid.org/00000002-7617-4539

Complete contact information is available at:

https://pubs.acs.org/10.1021/acs.jpcc.0c07613

\section{Author Contributions}

The manuscript was written through contributions of all authors. All authors have given approval to the final version of the manuscript.

\section{Notes}

The authors declare no competing financial interest.

\section{ACKNOWLEDGMENTS}

P.C. thankfully acknowledges the financial support from MINECO (ENE2016-74889-C4-2-R, AEI-FEDER-UE). E.P. and T.A. would like to thank the Leverhulme Trust (RPG2014-225). R.C.S. thanks the financial support from ANPCyT (PICT 2016-0679). L.M.A. thanks the KAUST Supercomputing Laboratory using the supercomputer Shaheen II for providing the computational resources.

\section{REFERENCES}

(1) Schreiber, F. Structure and Growth of Self-Assembling Monolayers. Prog. Surf. Sci. 2000, 65, 151-257.

(2) Vericat, C.; Vela, M. E.; Benitez, G.; Carro, P.; Salvarezza, R. C. Self-Assembled Monolayers of Thiols and Dithiols on Gold: New Challenges for a Well-Known System. Chem. Soc. Rev. 2010, 39, $1805-1834$

(3) Schwartz, D. K. Mechanism and Kinetics of Self-Assembled Monolayer Formation. Annu. Rev. Phys. Chem. 2001, 52, 107-137.

(4) Poirier, G. E. Coverage-Dependent Phases and Phase Stability of Decanethiol on $\mathrm{Au}(111)$. Langmuir 1999, 15, 1167-1175.

(5) Häkkinen, H. The Gold-Sulfur Interface at the Nanoscale. Nat. Chem. 2012, 4, 443-455.

(6) Torrelles, X.; Pensa, E.; Cortés, E.; Salvarezza, R.; Carro, P.; Hernández Guerrero, C.; Ocal, C.; Barrena, E.; Ferrer, S. Solving the Long-Standing Controversy of Long-Chain Alkanethiols Surface Structure on $\mathrm{Au}(111)$. J. Phys. Chem. C 2018, 122, 3893-3902.

(7) Pensa, E.; Cortés, E.; Corthey, G.; Carro, P.; Vericat, C.; Fonticelli, M. H.; Benítez, G.; Rubert, A. A.; Salvarezza, R. C. The Chemistry of the Sulfur-Gold Interface: In Search of a Unified Model. Acc. Chem. Res. 2012, 45, 1183-1192.

(8) Reimers, J. R.; Ford, M. J.; Halder, A.; Ulstrup, J.; Hush, N. S. Gold Surfaces and Nanoparticles Are Protected by $\mathrm{Au}(0)$-Thiyl Species and Are Destroyed When $\mathrm{Au}(\mathrm{I})$-Thiolates Form. Proc. Natl. Acad. Sci. 2016, 113, E1424.

(9) Maksymovych, P.; Sorescu, D. C.; Yates, J. T. Gold-AdatomMediated Bonding in Self-Assembled Short-Chain Alkanethiolate Species on the $\mathrm{Au}(111)$ Surface. Phys. Rev. Lett. 2006, 97, 146103.
(10) Guo, Q.; Li, F. Self-Assembled Alkanethiol Monolayers on Gold Surfaces: Resolving the Complex Structure at the Interface by STM. Phys. Chem. Chem. Phys. 2014, 16, 19074-19090.

(11) Pensa, E.; Carro, P.; Rubert, A. A.; Benítez, G.; Vericat, C.; Salvarezza, R. C. Thiol with an Unusual Adsorption-Desorption Behavior: 6-Mercaptopurine on $\mathrm{Au}(111)$. Langmuir 2010, 26, 17068-17074.

(12) Poirier, G. E.; Pylant, E. D. The Self-Assembly Mechanism of Alkanethiols on $\mathrm{Au}(111)$. Science 1996, 272, 1145.

(13) Darling, S. B.; Rosenbaum, A. W.; Wang, Y.; Sibener, S. J. Coexistence of the $(23 \times \sqrt{ } 3) \mathrm{Au}(111)$ Reconstruction and a Striped Phase Self-Assembled Monolayer. Langmuir 2002, 18, 7462-7468.

(14) Stettner, J.; Winkler, A. Characterization of Alkanethiol SelfAssembled Monolayers on Gold by Thermal Desorption Spectroscopy. Langmuir 2010, 26, 9659-9665.

(15) Qian, Y.; Yang, G.; Yu, J.; Jung, T. A.; Liu, G.-y. Structures of Annealed Decanethiol Self-Assembled Monolayers on $\mathrm{Au}(111)$ : An Ultrahigh Vacuum Scanning Tunneling Microscopy Study. Langmuir 2003, 19, 6056-6065.

(16) Nakaya, M.; Shikishima, M.; Shibuta, M.; Hirata, N.; Eguchi, T.; Nakajima, A. Molecular-Scale and Wide-Energy-Range Tunneling Spectroscopy on Self-Assembled Monolayers of Alkanethiol Molecules. ACS Nano 2012, 6, 8728-8734.

(17) Pensa, E.; Vericat, C.; Grumelli, D.; Salvarezza, R. C.; Park, S. H.; Longo, G. S.; Szleifer, I.; Méndez De Leo, L. P. New Insight into the Electrochemical Desorption of Alkanethiol Sams on Gold. Phys. Chem. Chem. Phys. 2012, 14, 12355-12367.

(18) Pensa, E.; Albrecht, T. Controlling the Dynamic Instability of Capped Metal Nanoparticles on Metallic Surfaces. J. Phys. Chem. Lett. 2018, 9, 57-62.

(19) Qian, H.; Jin, R. Ambient Synthesis of $\mathrm{Au}_{144}(\mathrm{SR})_{60}$ Nanoclusters in Methanol. Chem. Mater. 2011, 23, 2209-2217.

(20) Horcas, I.; Fernández, R.; Gómez-Rodríguez, J. M.; Colchero, J.; Gómez-Herrero, J.; Baro, A. M. Wsxm: A Software for Scanning Probe Microscopy and a Tool for Nanotechnology. Rev. Sci. Instrum. 2007, 78, No. 013705.

(21) Kresse, G.; Joubert, D. From Ultrasoft Pseudopotentials to the Projector Augmented-Wave Method. Phys. Rev. B 1999, 59, 17581775.

(22) Kresse, G.; Hafner, J. Ab Initio Molecular Dynamics for Liquid Metals. Phys. Rev. B 1993, 47, 558-561.

(23) Kresse, G.; Furthmüller, J. Efficiency of Ab-Initio Total Energy Calculations for Metals and Semiconductors Using a Plane-Wave Basis Set. Comput. Mater. Sci. 1996, 6, 15-50.

(24) Kresse, G.; Furthmüller, J. Efficient Iterative Schemes for Ab Initio Total-Energy Calculations Using a Plane-Wave Basis Set. Phys. Rev. B 1996, 54, 11169-11186.

(25) Blöchl, P. E. Projector Augmented-Wave Method. Phys. Rev. B 1994, 50, 17953-17979.

(26) Grimme, S. Semiempirical GGA-Type Density Functional Constructed with a Long-Range Dispersion Correction. J. Comput. Chem. 2006, 27, 1787-1799.

(27) Grimme, S.; Antony, J.; Ehrlich, S.; Krieg, H. A Consistent and Accurate Ab Initio Parametrization of Density Functional Dispersion Correction (DFT-D) for the 94 Elements H-Pu. J. Chem. Phys. 2010, 132, 154104.

(28) Pearson, W. B. A Handbook of Lattice Spacings and Structures of Metals and Alloys; Pergamon Press: 1958.

(29) Monkhorst, H. J.; Pack, J. D. Special Points for Brillouin-Zone Integrations. Phys. Rev. B 1976, 13, 5188-5192.

(30) Löfgren, J.; Grönbeck, H.; Moth-Poulsen, K.; Erhart, P. Understanding the Phase Diagram of Self-Assembled Monolayers of Alkanethiolates on Gold. J. Phys. Chem. C, Nanomater. interfaces 2016, 120, 12059-12067.

(31) Gao, J.; Li, F.; Zhu, G.; Yang, Z.; Lu, H.; Lin, H.; Li, Q.; Li, Y.; Pan, M.; Guo, Q. Spontaneous Breaking and Remaking of the RS$\mathrm{Au}-\mathrm{SR}$ Staple in Self-Assembled Ethylthiolate/Au(111) Interface. J. Phys. Chem. C 2018, 122, 19473-19480. 
(32) Holmes, S.; Palmer, R. E.; Guo, Q. Diffusion of $\mathrm{Au}\left(\mathrm{CH}_{3} \mathrm{~S}\right)_{2}$ on $\mathrm{Au}(111)$ Observed with the Scanning Tunneling Microscope. J. Phys. Chem. C 2019, 123, 24104-24110.

(33) Grönbeck, H.; Häkkinen, H. Polymerization at the Alkylthiolate- $\mathrm{Au}(111)$ Interface. J. Phys. Chem. B 2007, 111, 3325-3327.

(34) Carro, P.; Pensa, E.; Albrecht, T.; Salvarezza, R. C. Dynamics of RS-(Au-SR $)_{X}$ Staple Motifs on Metal Surfaces: From Nanoclusters to 2D Surfaces. J. Phys. Chem. C 2020, 124, 5452-5459.

(35) Munuera, C.; Barrena, E.; Ocal, C. Chain-Length Dependence of Metastable Striped Structures of Alkanethiols on $\mathrm{Au}(111)$. Langmuir 2005, 21, 8270-8277.

(36) Jiang, D.; Dai, S. Constructing Gold-Thiolate Oligomers and Polymers on $\mathrm{Au}(111)$ Based on the Linear S-Au-S Geometry. J. Phys. Chem. C 2009, 113, 7838-7842.

\section{NOTE ADDED AFTER ASAP PUBLICATION}

This paper was published on November 24, 2020. Due to production error, an author was listed with incorrect affiliations. The corrected version was reposted on November 25,2020 . 